\title{
N400-like responses to three-chord harmonic sequences with unexpected out-of-key endings: scalp topography, cortical sources, and perspectives for a clinical use
}

\author{
L. BONFIGLIO', A. VIRGILLITO', M. MAGRINI², A. PIARULLI ${ }^{3}$, M. BERGAMASCO ${ }^{3}$, \\ U. BARCARO ${ }^{2}$, B. ROSSI', O. SALVETTI', M.C. CARBONCINI' \\ ' Department of Translational Research on New Technologies in Medicine and Surgery, School of \\ Physical Medicine and Rehabilitation, University of Pisa, Pisa, Italy; ${ }^{2}$ Istituto di Scienza e Tecnologie \\ dell'Informazione "A. Faedo", Consiglio Nazionale delle Ricerche, Pisa, Italy; ${ }^{3}$ TeCIP Institute, \\ PERCRO Laboratory, Scuola Superiore Sant'Anna, Pisa, Italy
}

\begin{abstract}
A B S T R A C T
A series of ERP components, each provided with both a precise timing with respect to stimulation and a specific cortical localization, reflects the temporal succession of processing stages of music information. This makes the musical stimulus potentially usable to probe residual brain functions in non-communicating patients with disorders of consciousness. In an attempt to find a simple stimulation protocol that was suitable for use in a clinical setting, the purpose of this study was to verify whether a minimum-length musical stimulus, provided with a definite music-syntactic connotation, was still able to elicit musical ERPs in a group of eight healthy subjects. The stimulus was composed of the minimum number of chords necessary and sufficient to enable the subject to predict a plausible closure of the sequence (priming) and, at the same time, to provide him/her with the closing chord of the sequence (target), either congruous (probable closing) or not (improbable closing) to the tonal context. The subject's task was to discriminate and recognize the irregular targets. The components that were expected to be elicited, in this experimental situation, were ERAN, N5, P600/LPC. Conversely, in addition to these former components, we unexpectedly observed a N400-like component.

To determine whether this component was a real N400, we submitted our data to a sLORETA analysis in order to identify its cortical generators. Irregular chords showed higher current densities with respect to regular ones on the right-sided medial and superior temporal gyri, superior and inferior parietal lobules, fusiform and parahippocampal gyri, and on the bilateral posterior cingulate cortex. In particular, the N400-like wave seems to share with the word-primed music-elicited N400 certain generators that are located in cortical areas BA 21/37 and BA 22. This suggests that even chord-primed chord targets can convey extra-musical meanings and that, consequently, they might be useful in assessing residual higher-order information-processing capabilities in non-communicating patients with disorders of consciousness.
\end{abstract}

Key words

Music $\bullet$ Musical chords $\bullet$ Priming $\bullet N 400 \bullet$ Cortical sources $\bullet$ Consciousness

\section{Introduction}

"Appreciating and producing music simultaneously engage many complex perceptual, cognitive, and emotional processes, rendering music an ideal object for studying the mind" (Pearce and Rohrmeier, 2012). By paraphrasing and expanding the meaning of this sentence, we could say that music, precisely for its ability to activate simultaneously the multiplicity of brain functions that make up the 
human mind as a whole, is a potentially suitable tool to probe mind states and levels of awareness/ consciousness.

This concept becomes even more interesting if we consider the intimate connections between music and language. In fact, according to the Shared Syntactic Integration Resource Hypothesis (SSIRH) a non-domain-specific working memory system is involved in the integration of incoming elements (whether they are words or chords) within evolving structures (whether they are phrases or harmonic sequences) (Patel, 2008; Fedorenko et al., 2009). The partial overlapping and sharing of neural resources devoted to the processing of music and language explains also why children acquire information through prosodic features of language before they fully understand the meaning of words (Koelsch, 2011a). Therefore, it can be said that, especially in the early stages of life, language is rather analysed as a particular type of music (Koelsch, 2011a).

Thus, music is certainly one of the most fascinating topics of cognitive neuroscience and promises to have also positive effects on the clinical application of this discipline. In this context it is surprising that, to the best of our knowledge, music-elicited ERPs have never been used to date in the evaluation of residual higher-order information-processing capabilities of non-communicating patients with disorders of consciousness (DOC). This, despite musical stimuli have proven able to promote recovery in these patients (O'Kelly et al., 2013; Rollnik and Altenmüller, 2014).

\section{Music information processing and ERPs}

Brain processes involved in music perception are reflected, from a neuroelectric standpoint, in a series of event-related potential (ERP) components each provided with a precise timing in relation to the stimulus administration and with a specific cortical localization.

On this basis, therefore, a neurocognitive model of musical perception has been built which comprises a temporal succession of processing stages (brain computations) of music information coupled with specific neuroelectrical and, in part, anatomo-functional correlates (Koelsch, 2011a). Music-syntactic processing: ERAN and
P600/LPC

In any given chord progression whose mutual relationships follow the harmonic rules of the Western tonal system, music-syntactically irregular chord functions (i.e., out-of-key or dissonant chords) elicit an early negative potential between 150 and $350 \mathrm{~ms}$, which is located on frontal/fronto-temporal regions with right-hemispheric weighting (Early Right Anterior Negativity, ERAN) (Koelsch, 2011a). Those processes of music syntactic analysis that evoke ERAN include computations of both (a) the relation between a harmonic chord function and the global context of preceding chord functions (phrase structure grammar) and (b) the local transition probability from the penultimate to the final chord (finite state grammar) (Koelsch, 2011a). FMRI studies, during these operations, show the activation of the inferior frontal gyrus (IFG), pars opercularis (BA 44), with right-hemispheric weighting (Maess et al., 2001; Koelsch et al., 2002; Tillmann et al., 2003; Koelsch et al., 2005b; see also Koelsch, 2011a, for review).

When subjects attend to musical stimuli and detect structural incongruities, a late positive potential between 600 and $900 \mathrm{~ms}$ (P600 or Late Positive Component, LPC) is also evoked, which is localized on the posterior regions of the scalp (Janata, 1995; Koelsch, 2011a). This component is deemed to be linked to conscious detection processes of the musical-structural incongruities (Koelsch, 2011a) for both the permissive role of voluntary attention in regard to its generation and its temporal development, which has its maximum beyond the limit of $500 \mathrm{~ms}$. It seems to reflect processes of structural/ harmonic (re)integration and/or reanalysis and repair (Patel et al., 1998; Patel, 2003; Koelsch, 2011a) during the perception of rule-governed sequences. It is still debated whether such processes are related to context-updating and, therefore, P600/LPC is actually to be considered a later version of the P300/ P3b (Carrion and Bly, 2008; Koelsch, 2011a). In this case, its major neural sources would be located symmetrically in the parietal lobes (superior and inferior parietal lobule, postcentral gyrus and posterior cyngulate gyrus) with the contribution of other areas located in both the temporal (superior temporal gyrus) and occipital (fusiform gyrus and cuneus) lobes (Volpe et al., 2007; Wronka et al., 2012). 


\section{Musical meaning processing: N400 and N5}

The N400 is a characteristic ERP component pertaining to the linguistic domain. It appears approximately $400 \mathrm{~ms}$ after the presentation of a word (target) with an incongruous meaning with respect to the context (priming) of the sentence in which the target word has been inserted (Kutas and Federmeier, 2000, 2009 and 2011), thus reflecting semantic information processing. The $\mathrm{N} 400$ has a centro-parietal distribution and its amplitude varies in a direct relationship with the degree of inappropriateness of the closing word of the sentence (low cloze probability) and, consequently, with the degree of unpredictability of closure (Kutas and Federmeier, 2000, 2009 and 2011). The N400 effect is also observed when the target word follows a priming consisting in a musical excerpt (Koelsch et al., 2004), demonstrating that music is able to convey information that affects the semantic processing of words. Very short excerpts have been used as both prime (with word targets) and target (with word primes) (Daltrozzo and Schön, 2009a and 2009b): in both cases it has been possible to evoke a quite similar N400 with regard to latency, amplitude, and topography. In the music domain, therefore, the N400 reflects the processing of extra-musical meaning, with reference to the relationships between concepts conveyed by music and extra-musical world (words semantics) (Koelsch et al., 2004; Koelsch, 2011a and 2011b). Its cortical generators are bilaterally located in the posterior part of the Medial Temporal Gyrus (MTG) (BAs 21/37), in proximity of the superior temporal sulcus (STS) (Steinbeis and Koelsch, 2008a; Gallagher et al., 2014).

On the other hand, a later negativity (N5 or N500) in the time-window ranging from 450 up to over $800 \mathrm{~ms}$ and with a bilateral frontal distribution (Koelsch et al., 2000; Loui et al., 2005; Miranda and Ullman, 2007; Steinbeis and Koelsch, 2008b) is deemed to be associated with processing of the intra-musical meaning, that is, the meaning emerging from the reference of a musical element to another musical element, namely from the reciprocal (intra-musical) combinations of formal structures (Steinbeis and Koelsch, 2008b; Koelsch, 2011a and 2011b). Its cortical generators may be located both in the temporal lobe (possibly overlapping with those of the N400 in BAs 21/37) and in the frontal lobe (possibly in the posterior part of the inferior frontal gyrus) (Koelsch, 2011a).

\section{A new stimulus: theoretical assumptions on the basis of the Western tonal system}

Our study stems from the observation that in literature a wide variety of stimuli, often very complex, has been used to elicit music ERPs. This, to some extent, may have complicated the path toward an objective and unambiguous understanding of how musical stimuli are processed and analyzed by the brain. Therefore, in an attempt to identify a minimal musical stimulus, which still maintains its syntactic connotation, even though at a basic level, we used a sequence formed by the minimum number of chords necessary and sufficient to (i) insert the subject in a not ambiguous tonal context, so as to enable him/ her to predict (Bendixen, 2014) a plausible closure of the sequence (priming), and, at the same time, (ii) provide him/her with the closing chord of the sequence (target), either congruous (probable closing) or not (improbable closing or syntactic violation) to the previously determined tonal context.

We have therefore built up a new stimulus that obeys these criteria, taking into account two fundamental characteristics of the Western tonal system: (a) two chords represent the shortest sequence which is able to define a tonal context, when they are given, for example, by the dyad subdominant-dominant, and (b) the so-called "authentic cadence", consisting in the dyad dominant-tonic, is the natural, and virtually obligatory, ending of a tonal context.

It is immediately apparent that the common element to the two dyads (or syntagms, in the sense of structural syntactic units) is the dominant chord. In fact, this chord is the second of the early dyad, which identifies the tonal context and prepares the subject for the closure (first syntagm or prime), and, at the same time, the first of the later dyad, which forms the authentic cadence and closes the musical phrase (second syntagm or target).

By combining the two syntagms, therefore, it is possible to reduce the total number of chords by omitting one of the two dominant chords, which is clearly redundant, while respecting at the same time the correctness of syntactic rules that govern their mutual relationships, so that the musical phrase is syntactically correct and maintains its accomplished meaning. In this way, the two syntagms are joined together through the single dominant chord, which 
constitutes a sort of hinge between them. In the applied protocol, the third (and last) position of the sequence (i.e., the target position) is left free to be alternately occupied either by the tonic chord, which by completing the authentic cadence closes the sequence congruously (regular target/expected chord), or by a dissonant chord, that in violation of the rules of the Western tonal system closes the sequence incongruously (irregular target/unexpected chord). In this way, a prime-target paradigm is configured, which refers to the tonal context (i.e., tonal context priming and expectancy violation).

\section{A classical experimental paradigm: the odd-ball task}

The selected protocol was a paradigm of discrimination and recognition of the irregular targets administered with a probability of 0.2 (odd-ball task) because, in the perspective of a strictly clinical use of the method, it would have allowed us to get as many possible ERP components (ERAN, N5 and P600/LPC) in a single study session, that is, the maximum amount of information with the least amount of time.

\section{Aims and scope}

The purpose of this study was to verify whether the particular kind of stimulus used, which was characterized, as said, by the extreme essentiality of the music-syntactic connotation, was still able to elicit complex musical ERPs. This was done in an attempt to identify, according to the wellknown principle of simplicity set out by William of Ockham in the 14th century (Ockham's razor) (see Fitzpatrick, 2014), the minimum chord sequence which was necessary and sufficient for the evocation of musical ERPs.

Our primary goal was to study the topographical distribution of the obtained ERP components in order to facilitate their identification and verify their putative correspondence with known components of the musical ERPs. The components that were expected to be elicited, on the basis of literature, were ERAN, N5, P600/LPC (see Koelsch, 2011a, for review). Conversely, in addition to these former components, we unexpectedly observed an
ERP component similar to the N400 with respect to morphology, latency and topographic distribution. To determine whether that component was a real N400, we submitted our data to a sLORETA analysis in order to both identify its cortical generators and verify their overlapping with those known to give rise to the $\mathrm{N} 400$.

\section{Material and methods}

\section{Participants}

Eight musically untrained (i.e., no formal musical training received) right-handed male subjects, aged 31-43 years (mean \pm standard deviation $[\mathrm{SD}]=36$ \pm 2 years), with no hearing impairments, took part in the study.

Written informed consent was obtained from each subject. The study was approved by the local ethics committee (Comitato Etico Sperimentazione del Farmaco, Azienda Ospedaliero-Universitaria Pisana) and all study protocols were in accordance with the Declaration of Helsinki.

\section{Stimuli}

Each subject listened to a series of 500 stimuli administered through headphones with an intensity of aproximatelly $70 \mathrm{~dB}$ SPL. Each stimulus was a three-chord sequence with a total length of 1050 ms. Each chord lasted $350 \mathrm{~ms}$ and consisted of four voices and three notes. The inter-stimulus interval was $950 \mathrm{~ms}$. Each recording section lasted $(500 \mathrm{x}$ $(1.050+0.950) \mathrm{s}=16 \mathrm{~min} 40 \mathrm{~s}$.

As shown in Fig. 1, the first chord of each stimulus was a subdominant chord and the second was a dominant. The key assumed randomly, with equal probabilities, the twelve possible values from $\mathrm{C} 4$ (i.e., the fourth $\mathrm{C}$ key on an 88-key piano keyboard) to B4. The third chord was either the consonant tonic chord (regular target) or the dissonant chord (irregular target). The dissonant chord consisted of the tonic and three notes in the octave above, sharper than the tonic by 13,14 , and 15 semitones, respectively (e.g., when the tonic was $\mathrm{C}$, the octave-above notes where $\mathrm{C \#}, \mathrm{D}$, and $\mathrm{D \#}$ ). An example of the stimulus can be heard at the following link: https:// www.youtube.com/watch?v=HW6xrzhdBaU\&featu re=youtu.be. 


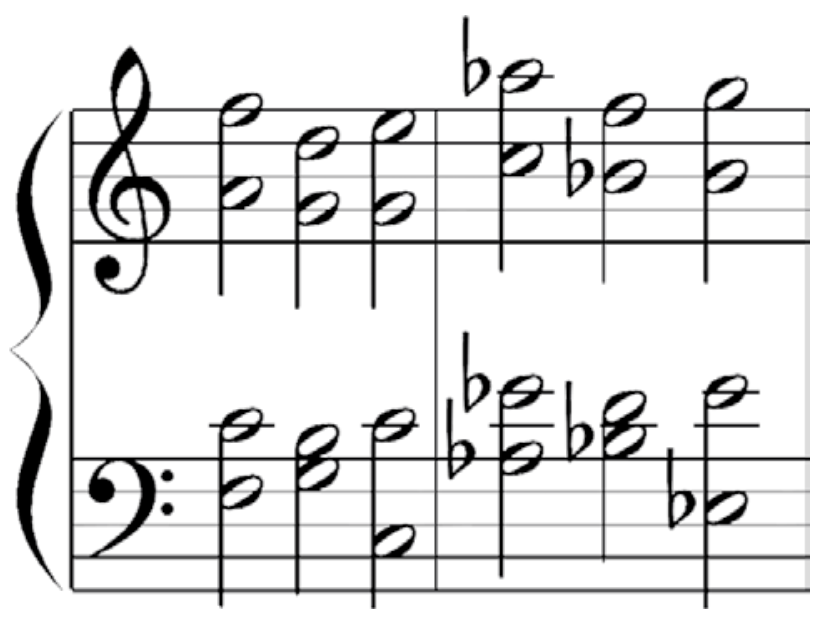

Fig. 1. - Two examples of subdominant-dominant-tonic chord succession. At left, tonic: C. At right, tonic: Eb.

\section{Experimental set}

During the recording session, the subject was seated on a chair in a noise-insulated room with a comfortable temperature.

According to the classical odd-ball paradigm, the occurrence probability of target stimuli was set so that the third chord was congruous with the tonal context in $80 \%(\mathrm{p}=0.8)$ and incongruous in $20 \%(\mathrm{p}$ $=0.2$ ) of cases. Subjects were not required to provide a judgment about the congruence of the irregular targets towards the tonal context, as in the classic relatedness judgement task (see below for details), but rather, given their strong syntactic unrelatedness to the context, only to count them silently, while ignoring the regular targets (odd-ball task). In this way, the subjects' attention was allocated mainly on the irregular targets, due both to their low occurrence probability (temporal context relevance) and to the need to provide a response (i.e., to take them into mental computation) (task relevance). At the end of each session, subjects were required to report the number of dissonant chords they had silently counted but, as a proof of the fact that irregular chords were easily identifiable, no omissions were detected.

\section{EEG recordings and pre-processing}

EEG signals were acquired using a BQ132S EEG amplifier (BrainQuick System, Micromed, Treviso, Italy) and an electrode cap (Electro-Cap International, Inc., Eaton, Ohio 45320 USA) with 19 predetermined recording locations (Fp1, Fp2, F8,
F4, Fz, F3, F7, T4, C4, Cz, C3, T3, T6, P4, Pz, P3, $\mathrm{T} 5, \mathrm{O} 1, \mathrm{O} 2)$ according to the 10-20 International System. The ground electrode was placed between $\mathrm{Fz}$ and $\mathrm{Fp} 1-\mathrm{Fp} 2$. Electrode impedance was always kept below $5 \mathrm{~K} \Omega$. EEG signals were digitised at a sampling rate of $256 \mathrm{~Hz}$ and band-pass filtered between 0.5 and $45 \mathrm{~Hz}$.

In line with previous papers from our group (Bonfiglio et al., 2013 and 2014; Carboncini et al., 2014), surface potentials were referenced offline (after ICA pruning) to an estimated infinity reference (IR) obtained by the reference electrode standardization technique (REST) (Qin et al., 2010), thereby rendering EEG data reference-free (Kayser and Tenke, 2010). The IR was chosen as this technique, by providing an approximate neutral/zero reference, has been demonstrated not to introduce any significant change of ERP features (including waveform and polarity) unlike other commonly used referencing schemes (Yao et al., 2007).

In addition, we also employed the average-mastoid reference (AMR) (i.e., the algebraic mean of the mastoid electrodes), which is commonly used in studies devoted to N400 recording, to verify if the N400-like component observed in this study still remained visible even with this referencing scheme. Eye movements were monitored by means of a pair of electrodes arranged diagonally to the horizontal line passing by the outer corners of the eyes, one electrode being placed $1 \mathrm{~cm}$ below the outher canthus of the left eye and the other one $1 \mathrm{~cm}$ above the outher canthus of the right eye (Bonfiglio et al., 2013 and 2014).

\section{Data analysis}

\section{Event-Related Potentials}

EEG epochs were analyzed in the time-domain and the corresponding time-locked average potential was extracted for each subject. The anchor-time point was set at the beginning of the third chord of the sequence.

The EEG signal was filtered between 0.5 and 20 $\mathrm{Hz}$ (Bekinschtein et al., 2009). The value of the high-pass cutoff frequency allowed the signal to be detrended. For each subject, 500 EEG epochs were considered, each of which started at the beginning of the third chord and lasted $1 \mathrm{~s}$. For each subject, out of the 400 responses to the more frequent stimulus, 100 were randomly selected for analysis, thus 
equalizing their number to that of the responses to the deviant stimulus, in order to avoid introducing a difference in the signal-to-noise ratio of the averages. A first stage of artifact rejection was carried out in the "EEGLAB" environment (Makeig et al., 1996; Delorme and Makeig, 2004) by applying the "FastICA" algorithm (Hyvärinen and Oja, 2000) to all the 19 traces recorded from each subject. To identify artifacts possibly missed by the ICA method, a second stage consisted in the rejection of 20 out of the 100 responses on the basis of a recognition of large deviations, possibly due to artifacts, from the average amplitude. For this purpose, the maximum deviation from the average amplitude was computed for each trace, according to the mathematical expression $\Delta=\max _{n}\left\{\left(\left|f_{n}\right|-\varphi\right) / \varphi\right\}$, where $\varphi$ stands for the average of the absolute values If $_{n} \mid$ of the signal values $f_{n}$. The response that included the trace signal presenting the maximum value of $\Delta$ was then iteratively rejected.

For each subject and each trace, the 80 retained responses to the more frequent stimulus and the 80 retained responses to the deviant stimulus were then averaged: therefore, for each subject, the calculated ERPs consisted of two waveforms (responses to the two kinds of stimulus) for each trace.

For presentation purposes only, averaged data were filtered with a $10 \mathrm{~Hz}$ low-pass filter (see Koelsch et al., 2004).

A statistical analysis was carried out independently from and preliminarily to the definite identification of the components, in order to identify which subepochs of the responses presented high statistical significance and to represent these sub-epochs in a visually effective way. For this purpose, for each trace, we applied the non-parametric Wilcoxon test to a moving time interval lasting $100 \mathrm{~ms}$, using the Matlab function "SignRank". In this way, it was possible to continuously assign to each instant (from 50 to $950 \mathrm{~ms}$ after the onset of the third chord) the value of the statistical significance (two-sided p-value) for the 100-ms interval centered on that instant.

\section{Scalp topography}

In order to obtain a general visualization of the topographic properties of the various components, we used the EEGLAB function "Topoplot" to obtain color maps of the scalp data field in a two-dimensional circular view together with equipotential lines. According to the color code, red corresponded to positive potentials and blue corresponded to negative potentials. The various images were obtained every $100 \mathrm{~ms}$ for both the responses to the deviant stimulus and those to the more frequent stimulus.

\section{Source analysis of the N400-like component}

For each subject and condition (regular and irregular targets), the cortical current density sources of the N400-like component were estimated within a timewindow encompassing the ERP peak (ranging from 375 to $425 \mathrm{~ms}$ after the stimulus onset) by means of sLORETA (Pascual-Marqui, 2002; Pascual-Marqui et al., 1994), which has been widely used to localize cortical current sources with set-ups consisting of as few as 19 electrodes (Bonfiglio et al., 2013 and 2014; Carboncini et al., 2014; Pascual-Marqui, 2002; Clemens et al., 2010).

Source localization in the time domain was performed on a three-shell spherical model (Montreal Neurological Institute brain atlas) registered to the Talairach brain atlas (Talairach and Tournoux, 1988). The model consists of 6,239 cortical grey matter voxels at $5 \mathrm{~mm}$ resolution. Electrode positions were registered to the spherical model following Towle and colleagues (Towle et al., 1993).

The statistical comparison between cortical current densities distributions (estimated at the latency of the N400-like component), related to irregular ('out-ofkey' chords) and regular targets ('in-key' chords), was conducted performing paired t-tests (between the two condition) for all voxels. The t-values significance was then estimated applying a single-threshold Statistical Non Parametrical Mapping (SNPM) approach (Nichols and Holmes, 2002), which takes into account the multiple comparison issue (simultaneous testing on all voxels) and at the same time does not require any assumption about the gaussianity of the tested distributions. SNPM works as follows: let us consider the null-hypothesis of no significant difference between the two conditions; under this null-hypothesis the labeling of the sLORETA maps for each individual can be changed randomly (i.e., the map pertaining to the congruous stimuli can be assigned to the incongruous stimuli or vice-versa). Based on this assumption, 200 random relabeling were made, and for each of them, the t-value related to each voxel test was extracted. For each relabeling, only the maximum t-value (in absolute value for two-tailed significance assessment) among 

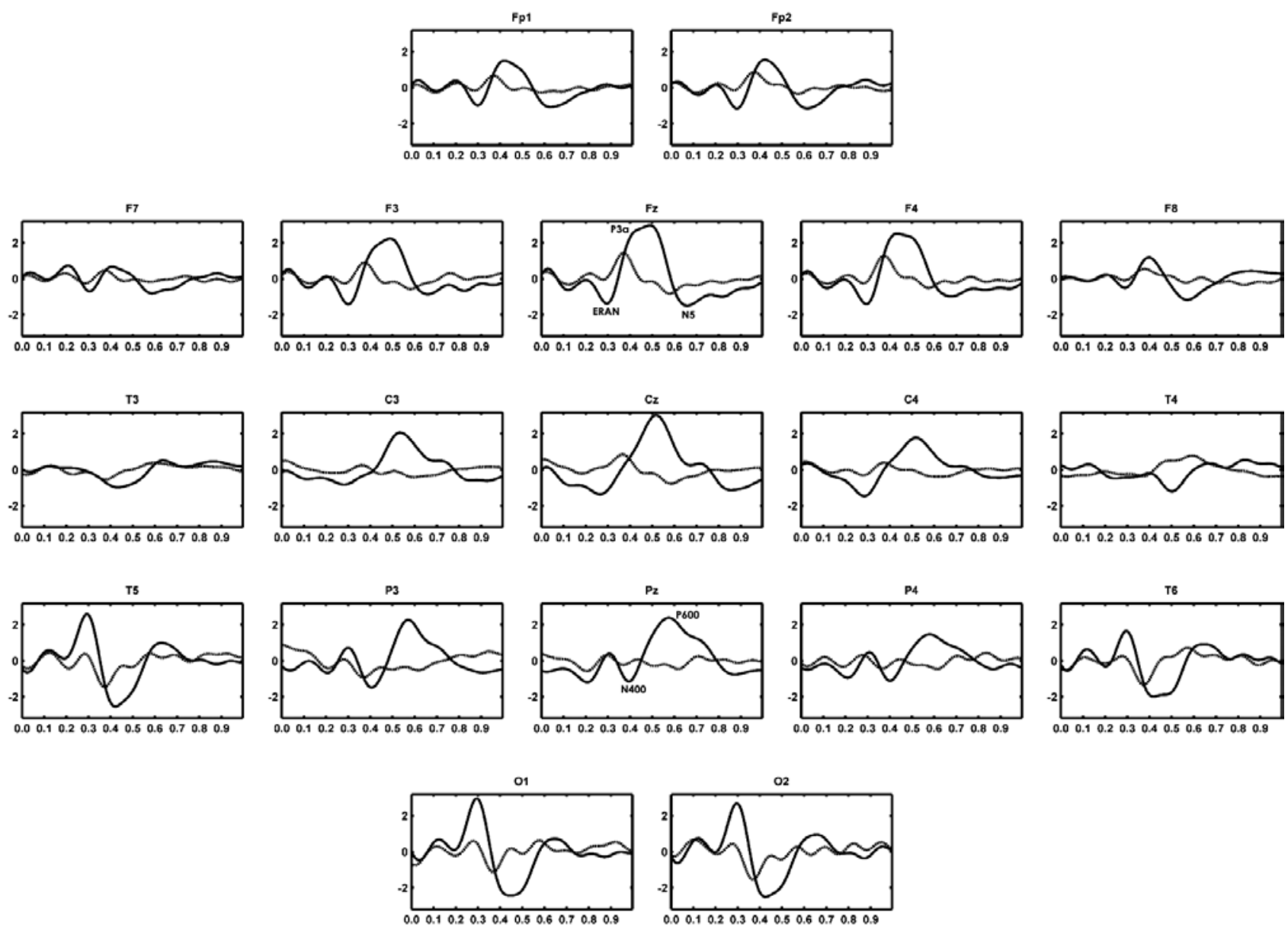

Fig. 2. - Grand-average ERPs elicited by three-chord harmonic sequences. Solid lines represent ERPs elicited by irregular targets ('out-of-key' chords), dashed lines represent ERPs elicited by regular targets ('in-key' chords). The $x$ axis shows the time in $\mathrm{ms}$, starting from the onset of the third chord. The $y$ axis shows the amplitude in $\mu \mathrm{V}$. Note that a negative peak at about $400 \mathrm{~ms}$ (N400-like wave) is clearly apparent on posterior sites (P3, Pz, P4, T5, T6, O1, O2).

voxels was retained. In this manner the maximum $\mathrm{t}$-values distribution under the null-hypothesis of no significant effect of the condition was extracted. The significance of each original t-value was then estimated as the ratio between the number of t-values of the null distribution exceeding the original t-value (in absolute value) and the number of relabeling. Here and in the following only p-values lower than 0.05 will be considered significant.

\section{Results}

\section{Event-Related Potentials}

As shown in Fig. 2, a sequence of five responses to dissonant chords could be identified, which we have labeled, with a criterion based solely on the detec- tion of both polarity and average latency, as either N100, P200, N300, P450, N650 in reference to the Fz site, or P100, N200, P300, N400, P600 in reference to the $\mathrm{Pz}$ site.

The first three components clearly showed a reversal of their polarity across the anterior and posterior regions of the scalp, while respective peak latencies remained substantially unchanged. This leads us to believe that they can be identified with the early components N1, P2 and N2 of the auditory ERP (which are classically labeled with a criterion based on both polarity and ordinal number), since the same polarity inversion between anterior and posterior regions of the scalp is known to occur with common average or infinite references with respect to average-mastoid references (Picton et al., 2000; Duncan et al., 2009. It should be noted, however, that in our recordings the anterior component labeled as N300 

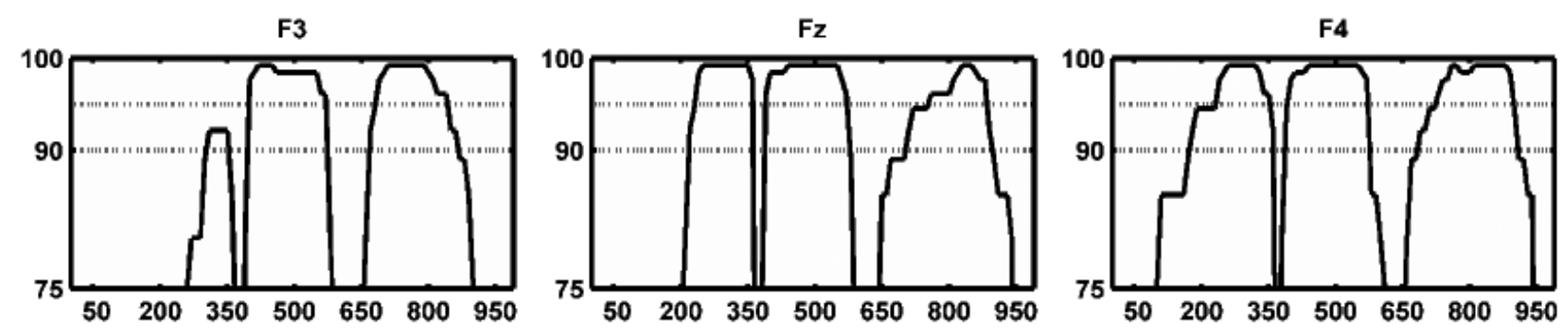

P3

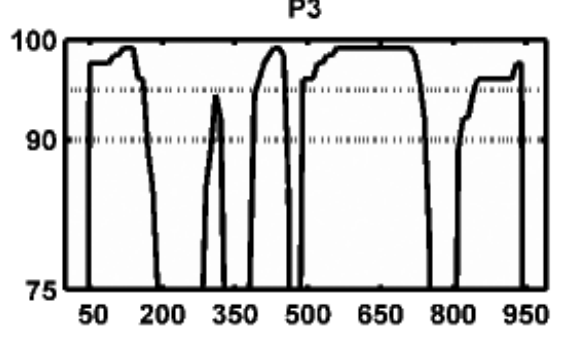

$\mathrm{Pz}$

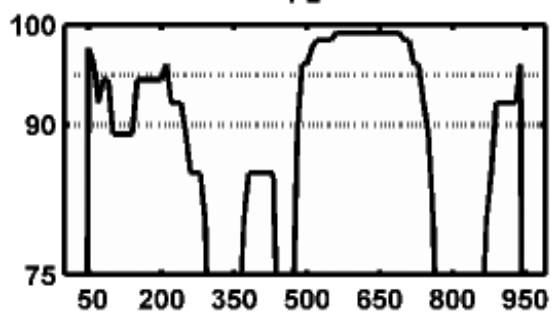

P4

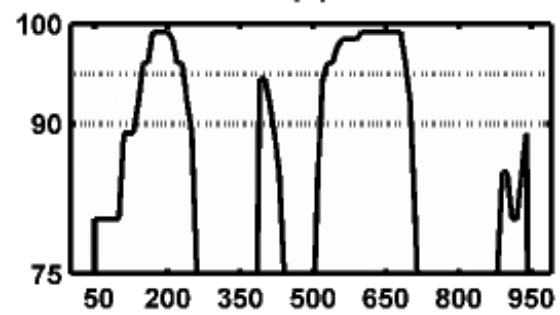

Fig. 3. - Statistical reliability of ERP components. Results of the non-parametric Wilcoxon test are depicted in relation to four representative recording sites (F3, F4, P3, P4). The $x$ axis shows the time in ms, starting from the onset of the third chord. The y axis shows the probability in percentage values given by the difference (1-p), $p$ being calculated using the Matlab SignRank function. The lower dotted line corresponds to a probability of $90 \%$, while the upper one to a probability of $95 \%$. Note that components showing the highest statistical significance are N300, P450 and N650, for anterior regions, and $\mathrm{N} 400$ and P600, for posterior regions.

was delayed with respect to the auditory N2(00), as well as the other following components, probably due to the need of musical stimuli to be processed in a more complex way than the simple tonal stimulus. The subsequent component, however, maintained its positive polarity across anterior and posterior regions of the scalp, but showed an anterior to posterior gradient of its peak latency: $450 \mathrm{~ms}$ at $\mathrm{Fz}, 500 \mathrm{~ms}$ at $\mathrm{Cz}$, and $600 \mathrm{~ms}$ at Pz. Such a behaviour is substantially similar to that of the P3(00) component of the auditory ERPs (Picton, 1992; Polich and Kok, 1995; Polich and Criado, 2006), so that we feel it reasonable to tentatively identify our anterior P450 with the P3a and our posterior P600 with the P3b of the auditory ERPs, in agreement with the view that considers the P600 component as part of the $\mathrm{P} 3 \mathrm{~s}$ family (see Carrion and Bly, 2008). Moreover, at $\mathrm{Pz}$ site, a negative wave appeared between the posterior components labeled as P300 and P600 with a peak latency at about 400 $\mathrm{ms}$, which, in the absence of any corresponding anterior component with reversed polarity, may be reasonably considered a stand-alone component with an original genesis from posterior regions. This component, which was a completely unexpected finding, seems to mimic a N400 response and will be hereafter referred to as a N400-like wave (Duncan et al., 2009).
As Fig. 3 shows, the continuous graphical representation of statistical significance offers a clear visual indication of the sub-epochs containing highly significant components. For instance, the graph for the F3 trace exhibits three separate intervals (approximately, 250-350 ms, 400-550 ms, 650-900 ms) and that for the P3 trace five (approximately, 50-200 ms, 250-350 ms, 380-450 ms, 500-750 ms and 800-950). Therefore, among all the components mentioned, those which have been the object of the present study (in particular, the anterior ones, corresponding to N300, P450, and N650, together with the posterior ones, corresponding to N400 and P600) were found to be statistically reliable.

It's worth noting that in all the recordings with an AMR (i) all five visible components at Fz site maintain their polarity also posteriorly and (ii) the N400like wave was never visible (Fig. 6).

\section{Scalp topography}

The study of the surface electric fields allows us not only to confirm the above mentioned correspondences but also to put forward new additional attributions, by keeping in mind that global topographic configurations are reference-independent 
FREQUENT
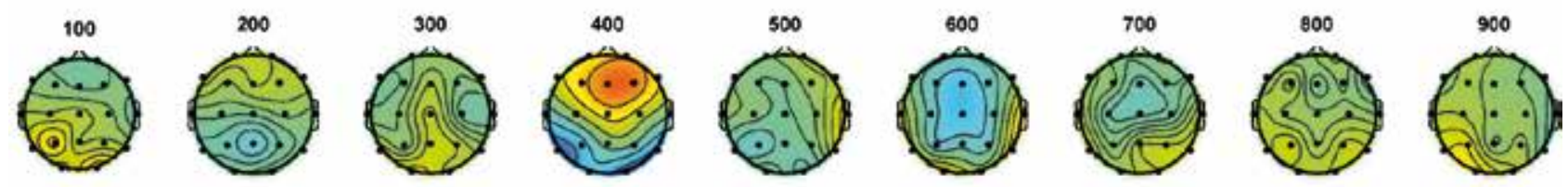

DEVIANT
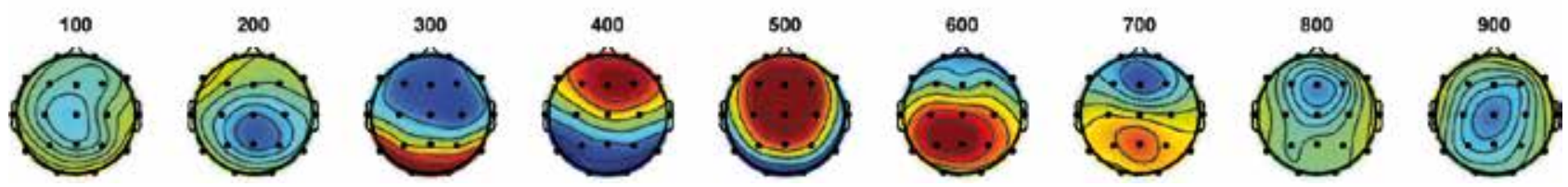

Fig. 4. - ERPs electric data fields, shown as isovoltage contour scalpmaps, for both frequent (regular/'in-key' chords, upper row) and deviant targets (irregular/ 'out-of-key' chords, bottom row). Note, in particular, the frontal negativity with right-weighting at around $300 \mathrm{~ms}$ (ERAN), the temporo-parietal-ocipital negativity at around $400 \mathrm{~ms}$ (N400-like wave), and the spatio-temporal progression of the P300-related fields from frontal (P3a) to centro-parietal regions (P3b or P600/LPC).

and directly linked to changes in configuration of the intracranial sources (Michel and He, 2011) (Fig. 4). In particular, the anterior component labeled as N300 was characterized by a frontal electric field with a right-hemisphere weighting. These topographic features, along with both polarity and latency, enable a probable identification of this component with the ERAN (Koelsch, 2011a).

Furthermore, the scalp distribution of the P3(00) components was confirmed (Bledowski et al., 2004; Polich, 2007; Wronka et al., 2012). They were characterized by a positive electric field that showed over time a spatial shift from frontal to posterior regions, with respective maxima temporally localized at about $450 \mathrm{~ms}$ on frontal regions, $500 \mathrm{~ms}$ on central regions, and $600 \mathrm{~ms}$ on parietal regions. This allows us to tentatively identify this latter component with the P600/LPC of the musical ERPs.

At around $400 \mathrm{~ms}$, an apparent dipole with a frontal positivity and a temporo-parieto-occipital negativity was visible, whose respective maxima, however, were not found to be superimposable in time, so as to make us hypothesize two separate components. In fact, the posterior maximum (labeled as N400 and possibly corresponding to the N400-like wave) anticipated the anterior one (labeled as P450 and possibily corresponding to the $\mathrm{P} 3 \mathrm{a}$ ) by about $50 \mathrm{~ms}$. In the time-window from 600 to $700 \mathrm{~ms}$, however, a dipole was visible with an anterior negativity and a posterior positivity, which partly overlapped the time course of P600/LPC. In a later time-window (from 700 to $800 \mathrm{~ms}$ ), a more sharply circumscribed medial frontal/fronto-central negative field seemed to emerge. The topographic distribution and peak latency of this component are both compatible with those of the N5 component of the musical ERPs (Miranda and Ullman, 2007; Steinbeis and Koelsch, 2008b).

\section{N400-like cortical sources}

ERAN, P600/LPC and N5 components which have been identified with reasonable certainty on the basis of their characteristics of polarity, latency and 


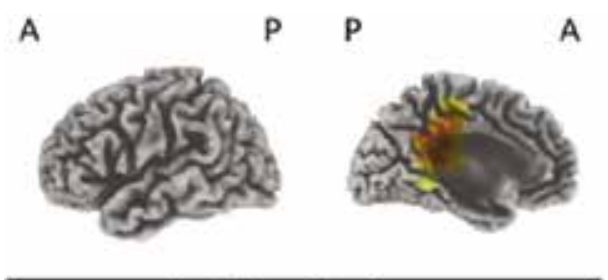

left hemisphere

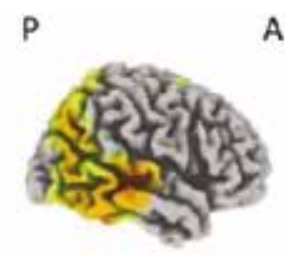

A

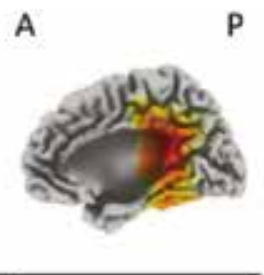

right hemisphere

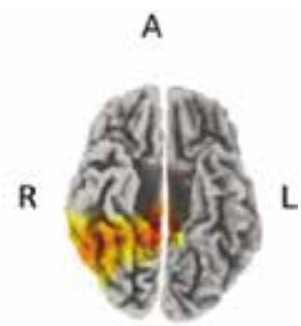

P

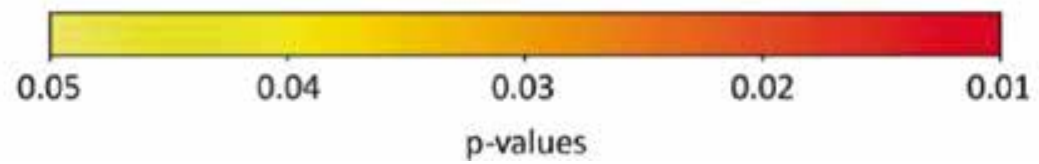

Fig. 5. - Statistical comparison between irregular ('out-of-key' chords) and regular targets ('in-key' chords) current densities in the N400-like wave time-window. Only voxels with a t-value corresponding to a p-value less than 0.05 are depicted ( $p<0.05$ corresponds to a t-value $>3.40$, while $p<0.01$ to a t-value $>4.58$ ). Yellow to red tones refer to progressively higher t-values. Throughout the figure, A denotes the anterior part of the cortex, $\mathrm{P}$ the posterior part, $\mathrm{R}$ the right hemisphere and $\mathrm{L}$ the left one. Note as irregular targets ('out-of-key' chords) show a higher current density with respect to regular ones ('in-key' chords) on the posterior part of both the upper and lower banks of the right superior temporal sulcus (STS), i.e., on both the right medial temporal gyrus (MTG) (BA 21/37) and the right superior temporal gyrus (STG) (BA 22), respectively. Additional cortical sources are detected on the right superior (SPL) (BA 7) and inferior parietal (IPL) lobules (BA 39/40), on the posterior cingulate (BA 23/31) and retrosplenial (BA 26/29/30) cortices, and on the right occipito-temporal, fusiform (BA 37) and parahippocampal (BA 34/35) gyri.

topographic distribution of the corresponding surface electric fields, correspond to the components we expected to obtain in this study considering the experimental paradigm employed.

On the contrary, the detection of a component similar to the N400 as regards its polarity, latency and topographic distribution, is a result that appears unexpected in the light of the literature on the topic. This led us to look for further evidence of its authenticity through the study of cortical current densities (see Fig. 5 and Table I). The main cortical areas showing significantly higher electrical activity (i.e., current density) in response to the irregular stimuli, when compared to the regular ones at the N400-like latency, were located in the posterior part of both the right medial temporal gyrus (BA 21/37) and the right superior temporal gyrus (BA 22), in proximity to the superior temporal sulcus, surprisingly in line, as will be discussed below, with what has been reported in literature regarding the true N400 cortical generators.

In addition, the elicitation of the N400-like wave was characterized, at cortical level, by significantly higher current densities (in comparison to no-elicitation) in the right superior (SPL) (BA 7) and inferior parietal (IPL) lobules (angular gyrus, BA 39, and supramarginal gyrus, BA 40), in the bilateral pos- terior cingulate (BA 23/31) and retrosplenial (BA 26/29/30) cortices, and, finally, in the right occipitotemporal, fusiform (BA 37) and parahippocampal (BA 34/35) gyri (i.e., in the parahippocampal cortex).

\section{Discussion}

\section{General findings}

In our opinion, the most important outcome of the present study lies in having shown that a syntactically basic musical stimulus (i.e., a minimum-length, although complete, chord sequence necessary and sufficient to drop the subject in an unambiguous tonal context, giving him/her at the same time the closing chord of the sequence) inserted in an oddball task, is able (a) to elicit statistically reliable musical ERPs whose morphology, latency and topographic distribution are consistent with those of the well-known components ERAN, N5 and P600/LPC, and, in addition, (b) is able to evoke a statistically reliable temporo-parietal negative component, with a peak latency of about $400 \mathrm{~ms}$, whose morphology, latency, topographic distribution, and cortical sources are superimposable to those of the N400 component. 


\begin{tabular}{|c|c|c|c|c|}
\hline & \multirow[t]{2}{*}{ Number of voxels ${ }^{a}$} & \multicolumn{3}{|c|}{ Number of activated voxels ${ }^{b}$} \\
\hline & & right & midline & left \\
\hline Middle Temporal Gyrus & 359 & $123(4.11)$ & & \\
\hline Precuneus & 355 & $89(4.51)$ & $6(4.12)$ & $3(3.88)$ \\
\hline Cingulate Gyrus & 287 & $51(4.64)$ & $20(4.42)$ & $25(4.11)$ \\
\hline Superior Temporal Gyrus & 419 & $93(4.15)$ & & \\
\hline Fusiform Gyrus & 231 & $80(4.06)$ & & \\
\hline Parahippocampal Gyrus & 185 & $59(4.21)$ & & $5(3.54)$ \\
\hline Superior Parietal Lobule & 134 & $53(4.05)$ & & \\
\hline Inferior Parietal Lobule & 286 & $41(3.96)$ & & \\
\hline Paracentral Lobule & 87 & $20(3.98)$ & $13(4.12)$ & $7(4.05)$ \\
\hline Posterior Cingulate & 87 & $26(4.58)$ & $5(4.40)$ & $9(4.11)$ \\
\hline Inferior Temporal Gyrus & 158 & $36(4.05)$ & & \\
\hline Medial Frontal Gyrus & 354 & $13(3.69)$ & $4(3.81)$ & $8(3.89)$ \\
\hline Supramarginal Gyrus & 55 & $20(3.81)$ & & \\
\hline Middle Occipital Gyrus & 145 & $16(3.82)$ & & \\
\hline Insula & 209 & $15(3.91)$ & & \\
\hline Lingual Gyrus & 180 & $14(4.06)$ & & \\
\hline Transverse Temporal Gyrus & 36 & $14(3.88)$ & & \\
\hline Sub-Gyral & 57 & $12(4.42)$ & & \\
\hline Angular Gyrus & 27 & $10(3.91)$ & & \\
\hline Postcentral Gyrus & 363 & $7(3.52)$ & & \\
\hline Middle Frontal Gyrus & 494 & $5(3.86)$ & & \\
\hline Inferior Occipital Gyrus & 32 & $3(3.44)$ & & \\
\hline Cuneus & 273 & $1(3.54)$ & & \\
\hline
\end{tabular}

Cortical sources of the N40O-like wave and anatomo-functional correlations

The main cortical current density sources showing significantly higher activations in response to irregular stimuli, when compared to regular ones at the N400-like wave latency, were found to be localized at the posterior part of both the right medial temporal gyrus (MTG) (BA 21/37) and the right superior temporal gyrus STG (BA 22), in proximity to the superior temporal sulcus (STS). This, by taking in consideration the lower resolution capacity of sLORETA with respect to fMRI, is in line with what is reported in literature, where incongruous word targets appear to activate the right MTG and incongruous chord targets the right STG (Steinbeis and Koelsch, 2008a).
In the present study, other cortical areas were shown to contribute to the genesis of the N400-like wave, whose activation seems to account for some aspects of the cognitive processing of the irregular chord targets used.

The activation of the right superior (SPL) (BA 7) and inferior parietal (IPL) lobules (angular gyrus, BA 39, and supramarginal gyrus, BA 40), for example, might be related to attentional orientation phenomena (attentional shift) triggered by the upcoming stimulus (Behrmann et al., 2004), while the activation of the bilateral posterior cingulate (BA 23/31) and retrosplenial (BA 26/29/30) cortices might be related to the conscious awareness of the stimulus (Raichle et al., 2001; Gusnard and Raichle, 2001).

Moreover, the activation of the right occipitaltemporal, fusiform (BA 37) and parahippocampal 

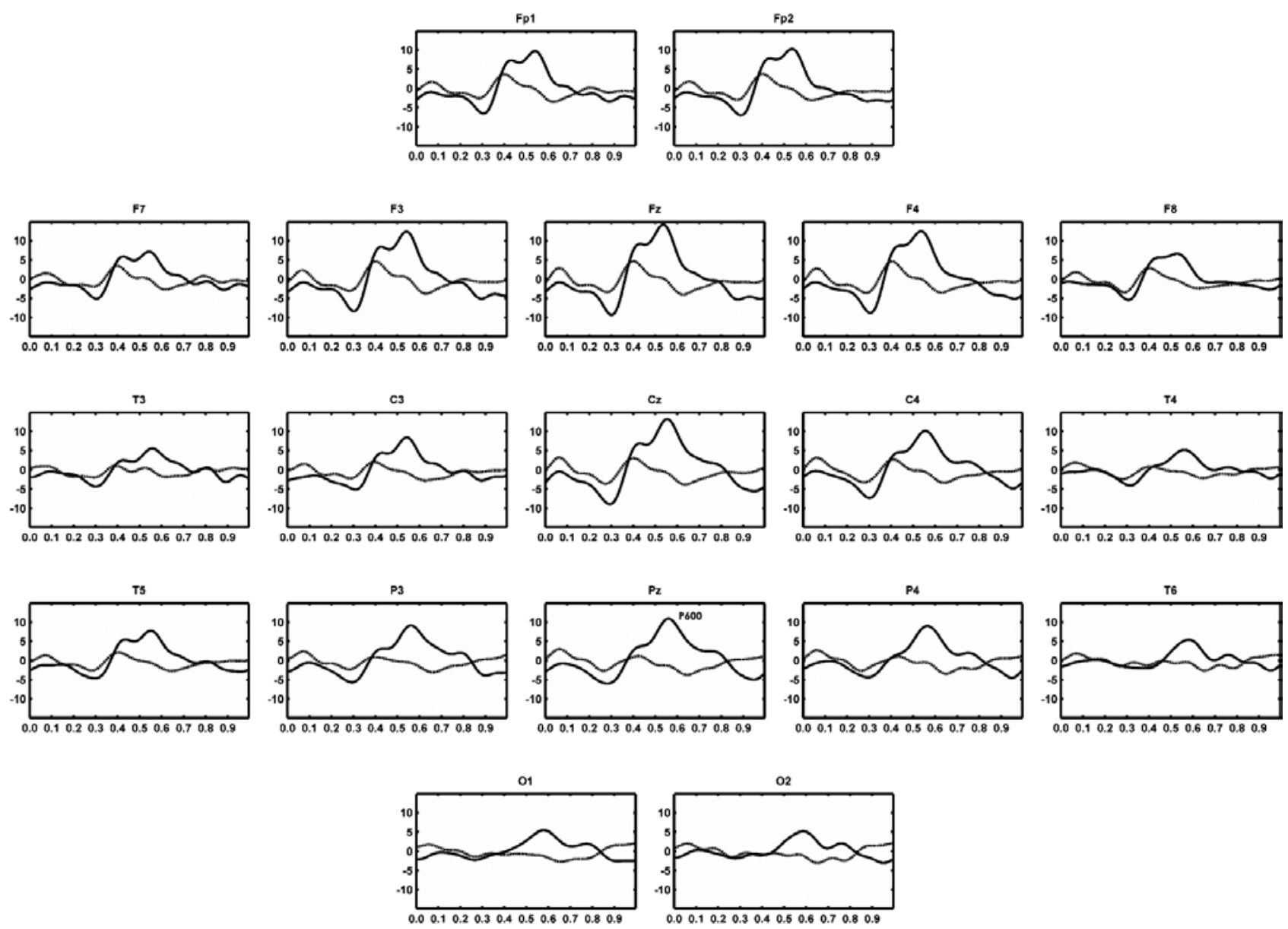

Fig. 6. - ERPs from a single representative subject recorded with an average-mastoid reference (AMR). Note that, with this referencing scheme, the N400-like wave is not visible. In the same temporal window of the N400-like wave, a small positive bump can be recognized on the ascending slope of the P600/LPC complex.

(BA 34/35) gyri (i.e., the parahippocampal cortex), although commonly attributed to visual information processing, has been recently linked to the processing of paralinguistic elements of verbal communication (Rankin et al., 2009; Aminoff et al., 2007 and 2013), that is, complex auditory information concerning 'pseudo-musical' characteristics of language (for example, prosody, intonation, etc.).

Finally, it is well-known that the parahippocampal cortex is involved in the cognitive processing of emotional/autonomic responses whether pleasant or unpleasant (Meerwijk et al., 2013; Koelsch, 2005a). The activation of this brain region is therefore fully consistent with the processing of the negative emotional valence conveyed by dissonant chords. It is a common experience, in fact, that hearing a "wrong/ false note' evokes an unpleasant feeling as a result of the disappointment of an expectation.

\section{A novel prime-target pair: chord prime in combination with chord target}

We have already mentioned, in the Introduction, the specific nature of the stimulus used in this study, that is its feature of simplicity in the sense of Occam's criterion. Our guiding principle in the construction of the stimulus was to obtain a musical sequence that contained both the minimum information necessary to make a closure prediction (prime) and the closure itself (target), be it congruous (regular target) or incongruous (irregular target) with respect to the preceding sequence. All this had to be included in a basic (i.e., simple) syntactic structure with accomplished meaning (prime-target pairs).

In the classical paradigm used to evoke the N400, the target stimuli (irregular and regular) are equiprobable (i.e., balanced condition), that is they have an occurrence probability of 0.5 (Koelsch, 2011a). The subject is asked to judge the relatedness of 
each stimulus with respect to the prime with twoalternative forced choice ("fits", "does not fit") (relatedness judgment task) (Koelsch, 2011a). Until now, to investigate the ability of music to convey extra-musical meanings, musical targets (consisting of $\sim 1 \mathrm{~s}$ short musical excerpts, single chords or even tones) have been normally used in combination with word primes (Daltrozzo and Schon, 2009a; Steinbeis and Koelsch, 2008a and 2011; Grieser-Painter and Koelsch, 2011).

In the present study, however, as the irregular target was strongly unrelated to the tonal context (i.e., totally unambiguous), we adopted a classic odd-ball task. In this paradigm, target stimuli are not equiprobable (i.e., unbalanced condition), that is irregular targets are presented with an occurrence probability of 0.2 (deviant-infrequent targets) and regular targets with a probability of 0.8 (standard-frequent targets). The subject was asked to count silently the irregular targets whenever they occurred (i.e., he/she had to hold mental computation of the irregular targets). To the best of our knowledge this is the first time that chord targets used in combination with chord primes have given rise to a N400-like wave.

By comparing the odd-ball task and the two-way relatedness task in terms of both attentional resources allocation and involved predictive activities, and by considering the particular type of target stimulus used in the present study, we analyze below those putative factors, pertaining to the experimental conditions adopted, that may have contributed to the generation of the N400.

\section{Attentional resources}

The unbalanced condition by itself makes the infrequent target (i.e., 'out-of-key' chord) a stimulus that emerges to the attention of the subject (i.e., with a bottom-up mode) only by virtue of the temporal-context regularity violation (Picton, 1992). In addition, it embodies a deviation from the tonal context regularity and, consequently, its contextual relevance (or salience) further increases. This irrespective of the fact that the required task is passive or active.

In an active odd-ball paradigm, the irregular target is not only relevant to the context, by virtue of its connotation of deviant-infrequent stimulus, but it also becomes a significant stimulus with respect to the task (task-relevant). That is, it represents precisely that change of the context that the subject has the task to detect (attended stimulus) and upon which, therefore, focuses his/her attention (i.e., with a topdown mode) (Polich and Criado, 2006).

The frequent target ('in-key' chord), however, by diluting in the context regularity (i.e., the background), becomes both context- and task-irrelevant. In a word, it will be almost completely ignored by the subject (unattended stimulus) (Picton, 1992).

The attentional load of the irregular target in this way is maximized, whilst that of the regular target is minimized. The end result is the strengthening of the attentional focus upon the irregular targets.

On the contrary, in the balanced condition regular and irregular targets are both relevant to the task. It follows that the subject top-down attentional resources are equally allocated on the processing of regular targets, leaving to the irregular target just the tonal-context relevance as a distinguishing feature (bottom-up mode).

It is reasonable to assume that the choice of an oddball paradigm, in this study, could have favored the N400 elicitation by narrowing and strengthening the subject's attentional focus exclusively on the irregular targets. It is known, in fact, that the N400 component shows a clear sensitivity to the attentional load manipulation (Kutas and Federmeier, 2009; Coull, 1998; Holcomb, 1988).

\section{Cloze probability}

We know that the amplitude of the linguistic N400 is much larger when the target word is incongruous and, as such, completely 'unpredictable' (low cloze probability) with respect to the sentence in which it is inserted, so as to give rise to a strong reaction of 'surprise' (Federmeier et al., 2007).

But, to what extent in our study is the irregular target inappropriate? The chord used can be rightly considered as doubly incongruous, both because it is 'out-of-key' with respect to the tonal context and because it lies outside the options offered by the Western tonal system. By analogy with linguistics, we might say that it is not part of the lexicon of the Western tonal system. This entails a violation not only of the combination rules of chords (phrasal or syntactic level), but also of the formation rules of chords (lexical or semantic level). We could call the irregular chord, in this sense, a 'pseudo-word'. It is reasonable to assume, therefore, that it represents 
the maximum possible incongruence with respect to the prime and, therefore, being provided with the lower cloze-probability, it is a particularly suitable stimulus for the N400 evocation.

\section{Extra-musical meaning/Semantics}

It has long been known that the N400 reflects the processing of extra-musical meanings (musical semantics), since it can be elicited by either target words primed by musical stimuli or musical stimuli primed by target words (Koelsch et al., 2004; Daltrozzo and Schön, 2009a; 2009b). It cannot be excluded that, in the present study, the irregular target chord, although primed by chord stimuli, is actually endowed with an intrinsic semantic value. In fact, in our opinion, the irregular chord target, similarly to what happens for other types of musical stimuli, may be able to activate a representation of a concept with meaning, which, in the present case, due to the extreme degree of incongruence, would be that of 'wrong/false note'. In other words, it is possible that the 'out-of-key' chord is recognized as a 'wrong/false note' at a representational, intuitive, level.

\section{EEG recording reference}

An additional factor, of a purely technical nature (i.e., the type of reference used in the recording of such ERPs) may have enabled, in the present study, the recording of the N400-like wave. In fact, the posterior negative component at about $400 \mathrm{~ms}$ is not detectable with an AMR, but only with an IR, regardless of the paradigm used.

In the same time window, the anterior positivity, which is apparent irrespective of the applied rereferencing technique, is matched with a posterior negativity when using the IR.

Two different hypotheses can be drawn from these findings: (1) the posterior negativity at $400 \mathrm{~ms}$ is the polarity-reversed counterpart of the anterior P3a, which reverses its polarity posteriorly with IR, similarly to what happens for the earlier components N1, P2, $\mathrm{N} 2$ (that posteriorly become $\mathrm{P} 1, \mathrm{~N} 2, \mathrm{P} 2$, respectively); or, (2) the posterior negativity at $400 \mathrm{~ms}$ is a true $\mathrm{N} 400$ which reverses its polarity on the frontal regions, generating a wave peak misinterpreted as a P3a. In the latter case, however, one must admit that the possibility of recording a N400 exclusively depends, at least in our experimental conditions, on the use of the IR.
Two elements are in favor of the N400 hypothesis. Firstly, as we have seen, the cortical generators of this negativity at $400 \mathrm{~ms}$ correspond to those of the N400 wave (Steinbeis and Koelsch, 2008a) and not to those of the P3a wave (Bledowski et al., 2004; Volpe et al., 2007; Wronka et al., 2012). These results are even more reliable as sLORETA results are independent from the referencing technique applied to the EEG (Babiloni et al., 2014). Secondly, preliminary unpublished data from our laboratory suggest that the amplitude of both components (i.e., posterior negative and anterior positive) is modulated by the increasing attentional load that is obtained by administering in succession the relatedness judgement task and the odd ball task in their passive (negative task: bottom-up mode) and active variants (positive task: top-down mode). It is known that this property does not belong to the $\mathrm{P} 3 \mathrm{a}$, which is largely resistant to the attentional effects (Bekinschtein et al., 2009), whilst it is characteristic of the N400 wave, which on the contrary shows a clear dependence on attention (Kutas and Federmeier, 2009; Coull, 1998; Holcomb, 1988).

\section{Conclusions}

In our opinion, the stimulation mode proposed in this study could be both the easiest and the most time-saving way available to date for obtaining the maximum possible amount of information about the different processing steps of musical stimuli. In fact, using a single stimulation paradigm, this method has been proven as able to evoke different ERP components, pertaining putatively to both the syntactic and semantic domains, each subtending the activation of specific functional brain areas and implying different processing levels of musical information at different degrees of awareness/consciousness of the stimulus. In particular, as the music-elicited N400-like wave shares certain neural circuits with the linguistic $\mathrm{N} 400$, it could allow us to investigate these circuits even beyond a temporary or permanent inability of the subject to understand spoken language. It should be also noted that the stimulus used has been proven capable of engaging emotional responses, as is shown by the activation of the parahippocampal cortex. All this makes the method presented herein, similar to what happens for the linguistic N400 (Harrison and Connolly, 2013; Steppacher et al., 2013; Cruse et al., 
2014), particularly suitable for the bedside monitoring of non-communicative DOC patients, even if further confirmations are required for evaluating the intersubject and intertrial statistical reliability of the ERP components observed in our study on a higher number of cases (Cruse et al., 2014).

Moreover, before proceeding to the implementation stage of the project, cross-comparison studies between paradigms (relatedness judgment and oddball tasks) and their variants (passive and active conditions) are needed in order to (a) obtain confirmation of the N400-like wave amplitude modulation in response to growing attentional demands, and (b) verify if the unbalanced paradigm, because of how it is designed, does not render superfluous the verbal communication of task instructions, bypassing the need of the subject to understand the spoken message. In relation to this second point, it would be very important to understand whether or not the regularity violation of both tonal and temporal contexts is per se sufficient to mark the irregular chord as the attentional target, thereby making the paradigm as it were 'self-explaining' (pseudo-active paradigm) (see Signorino et al., 1995). In this case, our test could be also applied to non-communicative patients with impaired language comprehension due to severe brain injuries, helping to define their differential diagnosis with respect to DOC patients.

\section{References}

Aminoff E.M., Kveraga K., Bar M. The role of the parahippocampal cortex in cognition. Trends Cogn. Sci., 17(8): 379-390, 2013.

Aminoff E.M., Gronau N., Bar M. The parahippocampal cortex mediates spatial and nonspatial associations. Cereb. Cortex, 17(7): 1493-1503, 2007.

Babiloni C., Del Percio C., Lizio R., Marzano N., Infarinato F., Soricelli A., Salvatore E., Ferri R., Bonforte C., Tedeschi G., Montella P., Baglieri A., Rodriguez G., Famà F., Nobili F., Vernieri F., Ursini F., Mundi C., Frisoni G.B., Rossini P.M. Cortical sources of resting state electroencephalographic alpha rythms deteriorate across time in subjects with amnesic mild cognitive impairment. Neurobiol. Aging, 35(1): 130-142, 2014.

Behrmann M., Geng J.J., Shomstein S. Parietal cortex and attention. Curr. Opin. Neurobiol., 14(2): 212217, 2004.
Bekinschtein T.A., Dehaene S., Rohaut B., Tadel F., Cohen L., Naccache L. Neural signature of the conscious processing of auditory regularities. Proc. Natl. Acad. Sci. U.S.A., 106: 1672-1677, 2009.

Bendixen A. Predictability effects in auditory scene analysis: a review. Front. Neurosci., 8: 60, 2014.

Bledowski C., Prvulovic D., Hoechstetter K., Scherg M., Wibral M., Goebel R., Linden D.E.J. Localizing P300 generators in visual target and distractor processing: a combined event-related potential and functional magnetic resonance imaging study. J. Neurosci., 24(42): 9353-9360, 2004.

Bonfiglio L., Olcese U., Rossi B., Frisoli A., Arrighi P., Greco G., Carozzo S., Andre P., Bergamasco M., Carboncini M.C. Cortical source of blinkrelated delta oscillations and their correlation with levels of consciousness. Hum. Brain Mapp., 34: 2178-2189, 2013.

Bonfiglio L., Piarulli A., Olcese U., Andre P., Arrighi P., Frisoli A., Rossi B., Bergamasco M., Carboncini M.C. Spectral parameters modulation and source localization of blink-related alpha and low-beta oscillations differentiate minimally conscious state from vegetative state/unresponsive wakefulness syndrome. PLoS One, 9: e93252, 2014.

Carboncini M.C., Piarulli A., Virgillito A., Arrighi P., Andre P., Tomaiuolo F., Frisoli A., Bergamasco M., Rossi B., Bonfiglio L. A case of post-traumatic minimally conscious state reversed by midazolam: clinical aspects and neurophysiological correlates. Restor. Neurol. Neurosci., 32(6): 767-787, 2014.

Carrion R.E. and Bly B.M. The effects of learning on event-related potential correlates of music expectancy. Psychophysiology, 45(5): 759-775, 2008.

Clemens B., Bessenyei M., Fekete I., Puskás S., Kondákor I., Tóth M., Hollódy K. Theta EEG source localization using LORETA in partial epilepsy patients with and without medication. Clin. Neurophysiol., 121(6): 848-858, 2010.

Coull J.T. Neural correlates of attention and arousal: insights from electrophysiology, functional neuropimaging and psychopharmacology. Prog. Neurobiol., 55: 343-361, 1998.

Cruse D., Beukema S., Chennu S., Malins J.G., Owen A.M., McRae K. The reliability of the N400 in single subjects: implications for patients with disorders of consciousness. Neuroimage Clin., $\mathbf{4}$ : 788-799, 2014.

Daltrozzo J. and Schön D. Conceptual processing in music as revealed by $\mathrm{N} 400$ effects on words and musical targets. J. Cogn. Neurosci., 21(10): 18821892, 2009a. 
Daltrozzo J. and Schön D. Is conceptual processing in music automatic? An electrophysiological approach. Brain Res., 1270: 88-94, 2009 b.

Delorme A. and Makeig S. EEGLAB: an open source toolbox for analysis of single-trial EEG dynamics including independent component analysis. $J$. Neurosci. Methods, 134: 9-21, 2004.

Duncan C.C., Barry R.J., Connolly J.F., Fischer C., Michie P.T., Naatanen R., Polich J., Reinvang I., Van Petten C. Event-related potentials in clinical research: guidelines for eliciting, recording, and quantifying mismatch negativity, P300, and N400. Clin. Neurophysiol., 120: 1883-1908, 2009.

Federmeier K.D., Wlotko E.W., De Ochoa-Dewald E., Kutas M. Multiple effects of sentential constraint on word processing. Brain Res., 1146: 75-84, 2007.

Fedorenko E., Patel A., Casasanto D., Winawer J., Gibson E. Structural integration in language and music: evidence for a shared system. Mem. Cognit., 37: 1, 2009.

Fitzpatrick S. Simplicity in the philosophy of science. The Internet Encyclopedia of Philosophy, ISSN 21610002, http://www.iep.utm.edu/, November 24, 2014.

Gallagher A., Beland R., Vannasing P., Bringas M.L., Valdes Sosa P., Trujillo-Barreto N.J., Connolly J., Lassonde M. Dissociation of the N400 component between linguistic and non-linguistic processing: a source analysis study. World J. Neurosci., 4: 25-39, 2014.

Grieser-Painter J. and Koelsch S. Can out-of-context musical sounds convey meaning? An ERP study on the processing of meaning in music. Psychophysiology, 48: 645-655, 2011.

Gusnard D.A. and Raichle M.E. Searching for a baseline: functional imaging and the resting human brain. Nat. Rev. Neurosci., 2: 685-694, 2001.

Holcomb P.J. Automatic and attentional processing: an event-related brain potential analysis of semantic priming. Brain Lang., 35(1): 66-85, 1988.

Harrison A.H. and Connolly J.F. Finding a way in: a review and practical evaluation of fMRI and EEG for detection and assessment in disorders of consciousness. Neurosci. Biobehav. Rev., 37(8): 1403-1419, 2013.

Hyvärinen A. and Oja E. Independent component analysis: algorithms and applications. Neur. Netw., 13: 411-430, 2000.

Janata P. ERP measures assay the degree of expectancy violation of harmonic contexts in music. $J$. Cogn. Neurosci., 7(2): 153-164, 1995.
Kayser J. and Tenke C.E. In search of the Rosetta Stone for scalp EEG: converging on reference-free techniques. Clin. Neurophysiol., 121: 1973-1975, 2010.

Koelsch S., Gunter T., Friederici A.D., Schröger E. Brain indices of music processing: "nonmusicians" are musical. J Cogn Neurosci., 12(3): 520541, 2000.

Koelsch S., Gunter T.C., von Cramon D.Y., Zysset S., Lohmann G., Friederici A.D. Bach speaks: a cortical "language-network" serves the processing of music. Neuroimage, 17(2): 956-966, 2002.

Koelsch S., Kasper E., Sammler D., Schulze K., Gunter T.C., Friederici A.D. Music, language, and meaning: brain signatures of semantic processing. Nat. Neurosci., 7: 302-307, 2004.

Koelsch S. Investigating emotion with music: neuroscientific approaches. Ann. N. Y. Acad. Sci., 1060: 412-418, 2005.

Koelsch S., Fritz T., Schulze K., Alsop D., Schlaug G. Adults and children processing music: an fMRI study. Neuroimage, 25: 1068-1076, 2005.

Koelsch S. Toward a neural basis of music perception - a review and updated model. Front. Psychol., 2: 110, 2011a.

Koelsch S. Toward a neural basis of processing musical semantics. Phys. Life Rev., 8: 89-105, 2011 b.

Kutas M. and Federmeier K.D. Electrophysiology reveals semantic memory use in language comprehension. Trends Cogn. Sci., 12: 463-470, 2000.

Kutas M. and Federmeier K.D. N400. Scholarpedia, 4(10): 7790, 2009.

Kutas M. and Federmeier K.D. Thirty years and counting: finding meaning in the N400 component of the event-related brain potential (ERP). Аnпи. Rev. Psychol., 62: 621-647, 2011.

Loui P., Grent-'t-Jong T., Torpey D., Woldorff M. Effects of attention on the neural processing of harmonic syntax in Western music. Cogn. Brain Res., 25: 678-687, 2005.

Maess B., Koelsch S., Gunter T.C., Friederici A.D. Musical syntax is processed in the area of Broca: an MEG study. Nat. Neurosci., 4: 540-545, 2001.

Makeig S., Bell A.J., Jung T.P., Sejnowski T.J. Independent component analysis of electroencephalographic data. pp. 145-151. In: Touretzky D., Mozer M., and Hasselmo M. (Eds.) Advances in neural information processing systems Vol. 8. Cambridge MA, The MIT Press, 1996.

Meerwijk E.L., Ford J.M., Weiss S.J. Brain regions associated with psychological pain: implications 
for a neural network and its relationship to physical pain. Brain Imaging Behav., 7(1): 1-14, 2013.

Michel C.M. and He B. EEG mapping and source imaging. pp. 1179-1202. In: Schomer D.L. and Lopes da Silva F.H. (Eds.) Niedermeyer's electroencephalography. Philadelphia, Wolters Kluwer, 2011.

Miranda R.A. and Ullman M.T. Double dissociation between rules and memory in music: an eventrelated potential study. Neuroimage, 38(2): 331345, 2007.

Nichols T.E. and Holmes A.P. Nonparametric permutation tests for functional neuroimaging: a primer with examples. Hum. Brain Mapp., 15(1): 1-25, 2002.

O'Kelly J., James L., Palaniappan R., Taborin J., Fachner J., Magee W.L. Neurophysiological and behavioral responses to music therapy in vegetative and minimally conscious states. Front. Hum. Neurosci., 7: 884, 2013.

Pascual-Marqui R.D., Michel M.C., Lehmann D. Low resolution electromagnetic tomography: a new method for localizing electrical activity in the brain. Int. J. Psychophysiol., 18: 49-65, 1994.

Pascual-Marqui R.D. Standardized low resolution brain electromagnetic tomography (sLORETA): technical details. Methods Find. Exp. Clin. Pharmacol., 24(Suppl. D): 5-12, 2002.

Patel A., Gibson E., Ratner J., Besson M., Holcomb P. Processing syntactic relations in language and music: an event-related potential study. J. Cogn. Neurosci., 10: 717-733, 1998.

Patel A. Language, music, syntax and the brain. Nat. Neurosci., 6: 674-681, 2003.

Patel A. Music, language, and the brain. New York, Oxford University Press, 2008.

Pearce M. and Rohrmeier M. Music cognition and the cognitive sciences. Top. Cogn. Sci., 4: 468-484, 2012.

Picton T.W. The P300 wave of the human eventrelated potential. J. Clin. Neurophysiol., 9(4): 456479, 1992.

Picton T.W., Bentin S., Berg P., Donchin E., Hillyard S.A., Johnson R., Miller G.A., Ritter W., Ruchkin D.S., Rugg M.D., Taylor M.J. Guidelines for using human event-related potentials to study cognition: recording standards and publication criteria. Psychophysiology, 37: 127-152, 2000.

Polich J. Updating P300: an integrative theory of P3a and P3b. Clin. Neurophysiol., 118(10): 2128-2148, 2007.
Polich J. and Criado J.R. Neuropsychology and neuropharmacology of $\mathrm{P} 3 \mathrm{a}$ and $\mathrm{P} 3 \mathrm{~b}$. Int. J. Psychophysiol., 60: 172-185, 2006.

Polich J. and Kok A. Cognitive and bilogical determinants of P300: an iintegrative review. Biol. Psychol., 41: 103-146, 1995.

Qin Y., Xu P., Yao D. A comparative study of different references for EEG dafault mode network: the use of the infinity reference. Clin. Neurophysiol., 121: 1981-1991, 2010.

Raichle M.E., MacLeod A.M., Snyder A.Z., Powers W.J., Gusnard D.A., Shulman G.L. A default mode of brain function. Proc. Natl. Acad. Sci. USA, 98: 676-682, 2001.

Rankin K.P., Salazar A., Gorno-Tempini M.L., Sollberger M., Wilson S.M., Pavlic D., Stanley C.M., Glenn S., Weiner M.W., Miller B.L. Detecting sarcasm from paralinguistic cues: anatomic and cognitive correlates in neurodegenerative disease. Neuroimage, 47(4): 2005-2015, 2009.

Rollnik J.D. and Altenmüller E. Music in disorders of consciousness. Front. Neurosci., 8: 190, 2014.

Signorino M., D'Acunto S., Angeleri F., Pietropaoli P. Eliciting P300 in comatose patients. Lancet, 345(8944): 255-256, 1995.

Steinbeis N. and Koelsch S. Comparing the processing of music and language meaning using EEG and fMRI provides evidence for similar and distinct neural representation. PLoS One, 3(5): e2226, 2008 a.

Steinbeis N. and Koelsch S. Shared neural resources between music and language indicate semantic processing of musical tension-resolution patterns. Cereb. Cortex, 18: 1169, 2008 b.

Steinbeis N. and Koelsch S. Affective priming effects of musical sounds on the processing of word meaning. J. Cogn. Neurosci., 23: 604-621, 2011.

Steppacher I., Eickhoff S., Jordanov T., Kaps M., Witzke W., Kissler J. N400 predicts recovery from disorders of consciousness. Ann. Neurol., 73(5): 594-602, 2013.

Talairach J. and Tournoux P. Co-planar stereotaxic atlas of the human brain Vol. 147. New York, Thieme, 1988.

Towle V.L., Bolanos J., Suarez D., Tan K., Grzeszczuk R., Levin D.N., Cakmur R., Frank S.A., Spire J.P. The spatial location of EEG electrodes: locating the best-fitting sphere relative to cortical anatomy. Electroencephalogr. Clin. Neurophysiol., 86: 1-6, 1993.

Tillmann B., Janata P., Bharucha J. Activation of the inferior frontal cortex in musical priming. Brain Res. Cogn. Brain Res., 16: 145-161, 2003. 
Volpe U., Mucci A., Bucci P., Merlotti E., Galderisi S., Maj M. The cortical generators of $\mathrm{P} 3 \mathrm{a}$ and $\mathrm{P} 3 \mathrm{~b}$ : a LORETA study. Brain Res. Bull., 73(4-6): 220230, 2007.

Wronka E., Kaiser J., Coenen A.M.L. Neural generators of the auditory evoked potential components P3a and P3b. Acta Neurobiol. Exp. (Wars), 72: 51-64, 2012.
Yao D., Wang L., Arendt-Nielsen L., Chen A.C. The effect of reference choices on the spatio-temporal analysis of brain evoked potentials: the use of infinite reference. Comput. Biol. Med., 37(11): 1529-1538, 2007. 